\title{
Trastornos de la conducta alimentaria en adolescentes de colegios públicos de Pereira
}

Moreno-Gutiérrez Paula Andrea ${ }^{1}$, Ochoa-Orozco Sergio Andrés ${ }^{1}$, Vásquez-Velásquez Diana ${ }^{2}$, Salazar-Becerra Diana ${ }^{2}$, Ortiz-Vanegas Erika ${ }^{2}$, López-Pantoja Yuli², Marín-Arango Lina ${ }^{2}$, García-Torres Adriana ${ }^{3}$

Médico. Universidad Tecnológica de Pereira. Pereira, Colombia.

Estudiante de Medicina, Universidad Tecnológica de Pereira. Pereira, Colombia.

Nutricionista y dietista. Docente del departamento de Medicina Comunitaria. Facultad Ciencias de la Salud. Universidad Tecnológica de Pereira. Pereira, Colombia.

Correspondencia: paula.rubicon@gmail.com

Fecha de Recepción: 18/04/2015

Fecha de Evaluación: 15/05/2015

Fecha de Solicitud de Correcciones: 18/11/2015

Fecha de Aceptación: 23/02/2016

\section{Resumen}

Introducción: Los trastornos de la conducta alimentaria (TCA) son la tercera enfermedad crónica más prevalente en adolescentes, por lo que se consideran un problema de salud pública.

Objetivo: Determinar la prevalencia de trastornos de la conducta alimentaria en estudiantes de media académica de colegios públicos de la zona urbana de la ciudad de Pereira

Métodos: Se realizó un estudio descriptivo con muestreo aleatorio mediante distribución proporcional entre los colegios públicos de la zona urbana de Pereira agrupados por sectores según su ubicación. Se aplicó la Encuesta de Comportamiento Alimentario (ECA).

Resultados: Se encuestaron 382 estudiantes, 58,9\% hombres. La ECA fue positiva en el $24,3 \%$ de los encuestados. Según el Índice de Masa Corporal el 9,7\% de la población se encontraba en rangos de delgadez, 5,8\% sobrepeso y $1,6 \%$ obesidad. Se encontró relación $(\mathrm{p}<0,05)$ entre el género femenino y la ECA positiva $(\mathrm{RM}=3,83)$, realización de dietas $(\mathrm{RM}=1,55)$, alteración de la auto imagen $(\mathrm{RM}=1,79)$, uso de laxantes $(\mathrm{RM}=2,58)$ e inducción del vómito $(\mathrm{RM}=4,41)$. El 33,4\% reportó tener con alguna frecuencia episodios de consumo de grandes cantidades de alimentos acompañados de sensación de culpa.

Discusión: Existen alteraciones comportamentales relacionadas con trastornos de la conducta alimentaria en esta población, por lo que es necesario generar programas de prevención y promoción de los TCA enfocados para adolescentes.

Palabras clave: Trastornos de la conducta alimentaria, Psiquiatría del adolescente, Tamizaje masivo
Eating disordder among adolescents in public schools from Pereira

\section{Abstract}

Introduction: Eating disorders (ED) are the third most prevalent chronic disease among adolescents and they are considered a public health issue.

Objective: To determine the prevalence of eating disorders in students from $10^{\text {th }}$ and $11^{\text {th }}$ grade in public high schools at the urban area of Pereira city.

Methods: A descriptive study was conducted. Participants were randomly sampled using proportional allocation among public schools at the urban area of Pereira and the "Encuesta de Comportamiento Alimentario (ECA)" was applied.

Results: 382 students were interviewed, of which $58.9 \%$ were male. ECA test scores were positive for eating disorders in $24.3 \%$ of the study sample. According to the Body Mass Index, $9.7 \%$ of the population was classified as underweight, $5.8 \%$ were overweighed and $1.6 \%$ were obese. The presence of episodes of eating large amounts of food followed by guilt was reported in $33.4 \%$ of the cases. A statistically significant relationship was found $(\mathrm{p}<0.005)$ among female gender and positive ECA results $(\mathrm{OR}=3.83)$, dieting $(\mathrm{OR}=1.55)$, altered self-image $(\mathrm{OR}=1.79)$, laxatives use $(\mathrm{OR}=2.58)$ and induced vomiting $(\mathrm{OR}=4.41)$.

Discussion: Clearly, eating behaviors related to ED are already established in this population; therefore, prevention and promotion programs focused on teenager populations should be designed.

Key words: Eating disorders, Adolescent Psychiatry, Mass screening

\section{Introducción}

Los trastornos de la conducta alimentaria (TCA) se caracterizan por una alteración en la percepción corporal, la cual es somatizada en signos y síntomas relacionados con los hábitos alimentarios. Estos trastornos pueden ser de dos tipos: la anorexia nerviosa (AN) en la cual predomina un miedo intenso a la ganancia de peso, con rechazo a mantener este en los valores mínimos aceptables y la bulimia nerviosa (BN) que se caracteriza por episodios recurrentes de voracidad seguidos por conductas de purga. El diagnóstico de trastorno del comportamiento alimentario no especificados (TCA-NE) se aplica a quienes presentan alteraciones compatibles con un TCA sin cumplir los criterios definidos para los dos anteriores por el Manual de Diagnóstico y Estadística para Trastornos Mentales $(1,2)$.

Estos trastornos afectan principalmente personas entre los 10 y los 24 años (3) y son la tercera enfermedad crónica más prevalente en adolescentes (4), establecidos en el 1 al 4\% de esta población a nivel mundial y presentes como síndromes parciales en 5 a 13\% (5).

Actualmente los TCA son un problema de salud pública debido al aumento de su incidencia y sus graves consecuencias (4). Las complicaciones derivadas de los TCA afectan todos los sistemas corporales y pueden resultar mortales o irreversibles como en el caso de la osteoporosis, retardo en el crecimiento y cambios estructurales cerebrales (6). La mortalidad a largo plazo es de hasta el $20 \%$ en AN a 20 años, y de $5 \%$ a 5 años en BN, convirtiéndolos en los trastornos psiquiátricos con mayor mortalidad a nivel mundial, principalmente atribuible al suicidio (7). El diagnóstico suele ser tardío y se realiza generalmente en estadios avanzados de la enfermedad (8).

En Colombia, se desconoce la prevalencia real de estas enfermedades. Hasta el momento, existen reportes de poblaciones de universidades y colegios en ciudades como Bogotá, Bucaramanga, Cali y Tunja (9-18), que no permiten establecer cifras representativas, y en algunos casos con limitantes metodológicas identificadas (19). En estudiantes universitarios de la ciudad de Cali, la población con mayor riesgo de TCA se encontró entre los 15 y 19 años, con una prevalencia hasta del 45,3\% (12). 
Estos hechos han generado un creciente interés a nivel internacional y diversos llamados para contribuir con su diagnóstico y control por parte de la Organización Mundial de la Salud (5). En este artículo, se presentan los resultados de un estudio que se desarrolló en estudiantes de educación media de colegios públicos en la ciudad de Pereira, buscando caracterizar los hábitos alimentarios y realizando pruebas de tamización para determinar la prevalencia de sus trastornos.

\section{Materiales y métodos}

Se realizó un estudio descriptivo, con un muestreo aleatorio mediante distribución proporcional por sectores, que tomó como población los 9033 estudiantes de los grados 10 y 11 de jornada diurna matriculados para el año académico 2011 en los colegios públicos de la zona urbana de Pereira, Risaralda, Colombia. La muestra se calculó con el programa Epidat 3.1 utilizando un intervalo de confianza de $95 \%$, una $\mathrm{p}<0,05$ y una prevalencia esperada del $50 \%$. Se utilizó esta prevalencia esperada dado que el presente fue el primer estudio realizado en la ciudad y había pocos reportes en este grupo etario en el país.

Las instituciones educativas incluidas se encontraban agrupadas en ocho núcleos determinados por la Secretaría de Educación Municipal de Pereira según su ubicación geográfica. Se realizó una distribución proporcional de la muestra para cada sector de acuerdo a su representación porcentual en la población, y se seleccionó aleatoriamente un colegio por núcleo. Cada institución educativa proporcionó su listado de alumnos de los cursos correspondientes (décimo y undécimo), a partir de la cual se seleccionó aleatoriamente la unidad muestral previamente calculada mediante el programa Epidat 3.1 de manera individual para cada colegio, con una tasa de pérdida esperada del $50 \%$, debido a que se excluyeron los estudiantes menores de 15 años y en caso tal de que el estudiante seleccionado no hubiera ido ese día al colegio.

La recolección de la información se realizó con la encuesta de "Evaluación del Comportamiento Alimentario (ECA)" desarrollada y validada en Colombia para la tamización de TCA. Este instrumento tiene sensibilidad del 73,6 al $91,8 \%$ y especificidad entre 52,4 y $80,0 \%$; con un valor predictivo positivo entre 45,3 y $78,2 \%$ y uno negativo de 77,5 a $93,7 \%$, considerando un valor total mayor a 23 puntos como punto de corte para presencia de TCA según el método de tamización, de acuerdo a estudios realizados en Bogotá y Bucaramanga $(11,20)$. La encuesta incluye una serie de preguntas que evalúan la existencia de los diferentes criterios diagnósticos para los TCA, mediante el uso de frases afirmativas que describen comportamientos, hábitos, actitudes, conceptos e ideas acerca de la alimentación, la figura y peso corporal, así como los métodos de control de los mismos.

Este instrumento fue diseñado para ser auto aplicado por el participante, consta de 32 preguntas de las cuales 29 son de elección múltiple, de las cuales en 3 se pueden marcar de una a seis respuestas como afirmativas, con puntuación aditiva con una sola opción de respuesta y las demás son de única escogencia entre cuatro opciones, según las cuales se asignan de 0 a 3 puntos, siendo 0 y 1 comportamientos normales, 3 patológicos y 2 indeterminados. Las dos preguntas restantes miden la realización de conductas de purga (si/no) y en caso de ser afirmativa, se solicita la frecuencia del suceso.

La puntuación para las preguntas de opción múltiple es de 0 a 4, para las de negación o afirmación es de 0 o 1 y en las que aceptan múltiples opciones es de $0,3,5$ ó 6 . La suma de puntajes da un valor entre 0 y 94 (20). Además, la pregunta cuatro indaga los hábitos alimentarios del sujeto, incluyendo la frecuencia de los diferentes tiempos de comidas (desayuno, almuerzo, comida, meriendas, etc.).La encuesta fue diligenciada por los participantes, con indicaciones previas dadas por el grupo investigador y asesoría durante su diligenciamiento. Un equipo de 7 estudiantes de medicina previamente capacitados por un psiquiatra y una nutricionista fueron los encargados de la recolección de la información.

La medición de talla y peso de los participantes se realizó con dos pesas digitales previamente calibradas y un tallímetro de consultorio médico instalado en una pared de las instituciones educativas, previa estandarización del procedimiento, en todos los colegios este procedimiento fue practicado por la misma persona. La información se digitalizó en una base de datos utilizando Microsoft Office Excel 2013 y posteriormente fue analizada mediante el software SPSS 20. Se calculó el Índice de Masa Corporal (IMC) y de acuerdo a este se realizó la clasificación nutricional según los percentiles por edad y género para personas hasta los 20 años determinados por los Centros para el Control y Prevención de las Enfermedades (CDC).

Se calcularon frecuencias absolutas y medidas de dispersión para todas las variables. Se utilizó la pruebas estadística Chi Cuadrado de Pearson y se obtuvo Razón de Momios (RM) cruda para analizar la relación de entre dos variables dicotómicas o la prueba U de MannWhitnney para relacionar variables dicotómicas con cuantitativas discretas, considerando como significativos resultados con $\mathrm{p}<0,05$. Finalmente, se realizó una regresión logística binaria tomando como desenlace el riesgo de tener TCA (si/no) y como covariables el sexo, núcleo, estado nutricional según IMC (delgadez, normal y sobrepeso/ obesidad) y edad (15 a 17 años y 18 a 20 años).

Esta investigación fue aprobada por el comité de bioética de la Universidad Tecnológica de Pereira en la categoría de "investigación sin riesgo" el 13 de junio de 2011, según la resolución No. 8430 de 1993 del Ministerio de Salud de Colombia. Se obtuvo permiso escrito previo de cada una de las instituciones educativas, así como consentimiento informado por parte de los alumnos participantes y su respectivo tutor legal. Todo el proceso del estudio fue auto financiado por el equipo investigador e hizo parte del proceso formativo curricular del programa de Medicina de la Universidad Tecnológica de Pereira.

\section{Resultados.}

La muestra estuvo constituida por 382 estudiantes, el 58,9\% ( $\mathrm{n}=225)$ fueron hombres y el rango de edad se situó entre 15 y 20 años con media de 16 (DE: 1,1). El IMC promedio fue de $19,8 \mathrm{Kg} / \mathrm{m}^{2}$, variando de 15,4 a 39,8 (DE: 2,9) y según este, la mayoría de los adolescentes se encontraban en clasificación nutricional normal ( $82,5 \%, n=307), 9,9 \%$ $(n=37)$, de la población en rangos de delgadez, $5,8 \%(n=22)$ sobrepeso y $1,6 \%(n=6)$ obesidad, sin diferencia estadísticamente significativa según el género. Las características generales de las personas incluidas se muestran en el cuadro 1. 
Cuadro 1. Distribución de la muestra por núcleos geográficos de colegios, Pereira Colombia 2011.

\begin{tabular}{lccc}
\hline & $\mathrm{n}=382(\%)$ & $\%$ mujeres & $\begin{array}{c}\text { ECA } \\
\text { positiva (\%) }\end{array}$ \\
\hline Sexo (Mujer/Hombre) & $\begin{array}{c}225 / 157 \\
(41,1 / 58,9)\end{array}$ & - & $38,8 / 14,2$ \\
Edad & & & \\
15 a 17 años & $330(88,0)$ & 38,5 & 23,9 \\
18 a 20 años & $45(12,0)$ & 57,8 & 31,1 \\
Estado nutricional & & & \\
según IMC & & & \\
Delgadez & $37(9,9)$ & 32,4 & 16,2 \\
Normal & $307(82,5)$ & 42,0 & 24,7 \\
Sobrepeso u & $28(7,5)$ & 35,7 & 35,7 \\
obesidad & & & \\
Núcleo & & & \\
2 & $10(2,6)$ & 60,0 & 20,0 \\
3 & $147(38,5)$ & 23,1 & 17,0 \\
4 & $91(23,8)$ & 50,5 & 29,7 \\
5 & $15(3,9)$ & 40,0 & 13,3 \\
7 & $44(11,5)$ & 59,0 & 38,6 \\
8 & $75(19,6)$ & 52,0 & 26,6 \\
\hline
\end{tabular}

La ECA presento puntajes de 5 hasta 45 con promedio de 18 puntos (DE: 7,2). El 24,3\% ( $\mathrm{n}=93$ ) de la población presento positividad de la ECA, siendo este porcentaje más alto en mujeres ( $38,9 \%$ positivas, $\mathrm{n}=61$, mediana: 21 , rango: $8-45$, de: 8,1$)$ que en hombres $(14,2 \%$ positivos, $n=32$, mediana: 17 , rango: $5-35$, de: 5,9). Al realizar el análisis multivariado, la única variable relacionada con la positividad de la ECA fue el sexo femenino con una RM de 3,6 (IC95\% 2,13-6,11). Por otra parte, el $54,1 \%(n=203)$ de los entrevistados afirmaron consumir las 3 comidas básicas diarias (desayuno, almuerzo y cena) los 7 días de la semana. La cantidad de alimentos principales consumida semanalmente fue significativamente menor en las personas que presentaban ECA positiva $(\mathrm{p}<0,05)$. Los alimentos más consumidos durante la merienda fueron: cafetería y pastelería en un $36,9 \%$ de casos $(\mathrm{n}=141)$, seguidos por las bebidas no dietéticas $(33,8 \%$, $\mathrm{n}=129)$ y los dulces $(27,7 \%, \mathrm{n}=106)$, mientras el $9,7 \%$ de la población reportó consumir frutas $(n=37)$ y el $6,5 \%$ dijo consumir alimentos de cualquier clase $(n=25)$.

El consumo de alimentos en momentos de estrés emocional fue reportado "siempre" en el 7,9\% (n=30) de los estudiados, "frecuentemente" en el 11,5\% ( $\mathrm{n}=44) \mathrm{y}$, "a veces" en el 50,8\% ( $\mathrm{n}=194)$. La restricción de la alimentación o la realización de algún tipo de dieta para control de peso eran practicadas por el 33,8\% de las personas $(n=129)$, hecho relacionado con tener IMC elevado para la edad ( $<<0,001$. RM: 3,864. IC95\%: 1,726-8,649). Además, una cantidad similar de encuestados $(33,4 \%, \mathrm{n}=127)$ dijo tener con alguna frecuencia episodios de consumo de grandes cantidades de alimentos acompañados de sensación de culpa.

La autoimagen corporal fue concebida como adecuada o normal por el $61,5 \%,(n=235)$ mientras tanto el $26,7 \%(n=102)$ cree que puede ser mejorada con algunos cambios en la alimentación. El $3,1 \%$ de se considera obeso o con exceso de grasa $(n=12)$ y al $7,9 \%$ $(\mathrm{n}=30)$ restante le desagrada la forma de su cuerpo o parte de él. La existencia de algún grado de desagrado con la apariencia corporal esta estadísticamente ligada al género femenino (Cuadro 2), a tener IMC normal ( $p=0,003$. RM: 0,448 . IC95\% 0,260-0,773) y elevado ( $p=0,012$. RM: 2,671. IC95\% 1,212-5,883).

Cuadro 2. Relación estadística entre género femenino y variables incluidas en la Encuesta de Comportamiento Alimentario en estudiantes de colegios públicos de Pereira.

\begin{tabular}{llll}
\hline Variable & Valor de $\mathrm{p}^{*}$ & $\mathrm{RM}$ & $\mathrm{IC}$ \\
\hline Consumo de alimentos & 0,005 & 1,94 & $1,218-3,111$ \\
$\begin{array}{l}\text { en momentos de estrés } \\
\text { Auto imagen alterada }\end{array}$ & 0,006 & 1,79 & $1,179-2,741$ \\
$\begin{array}{l}\text { Inducción de vómito } \\
\text { Uso de laxantes }\end{array}$ & 0,001 & 4,41 & $1,698-11,461$ \\
Realización de dietas & 0,006 & 2,58 & $1,286-5,203$ \\
*Prueba de Chi cuadrado de Pearson. IC. Intervalo de confianza
\end{tabular}

*Prueba de Chi cuadrado de Pearson. IC: Intervalo de confianza

Como se muestra en la figura 1, las mujeres presentaban mayor riesgo que los hombres de consumo de alimentos en momentos de estrés, alteración de la auto imagen, inducción del vómito, uso de laxantes y realización de dietas. El consumo de cigarrillo fue reportado por el $3,2 \%$ de la población $(n=12)$ y el de alcohol por el $48,7 \%(n=186)$, siendo mayor este último hábito en hombres $(57,8 \%, \mathrm{n}=130)$ que en mujeres $(35,6 \%, n=56)(p<0,001$. RM: 2,468. IC95\% 1,621-3,757). El 29,4\% ( $n=33)$ de las mujeres reportaron tener alteraciones de sus ciclos menstruales y el $1,3 \%(\mathrm{n}=2)$ presentaban amenorrea por más de 3 meses en el momento de la encuesta, alteración relacionada con el uso de anticonceptivos ( $\mathrm{p}=0,009$. RM: 3,6. IC95\% 1,315-9,875).

Figura 1. Prevalencia de conductas alimentarias según el género en estudiantes de media académica de Pereira.

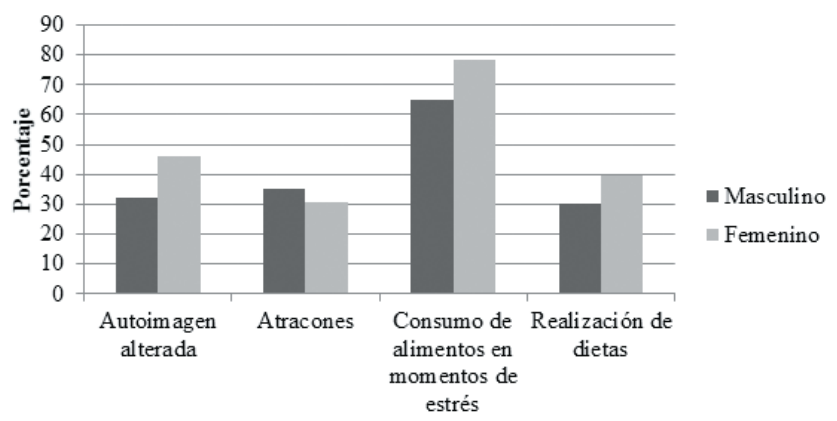

La inducción de vómito se presentó en el 10,8\% de las mujeres $(\mathrm{n}=17)$ y el $2,6 \%$ de los hombres $(n=6)$, el uso de laxantes se encontró en el $14,6 \%$ de las mujeres $(n=23)$ y $6,2 \%$ de los hombres $(n=14)$ (Figura 2$)$, los diuréticos fueron usados por el $2,9 \%$ de los encuestados $(n=12)$, con porcentajes similares para ambos géneros. No se encontró una relación significativa entre la realización de las conductas de purga y el IMC, mientras que estas conductas se encontraron relacionadas entre sí (inducción de vómito, uso de laxantes y uso de diuréticos) y con la realización de dietas, mostrándose que su coexistencia es frecuente (ver Cuadro 3). 
Figura 2. Conductas purgativas de acuerdo al género para estudiantes de media académica de Pereira.

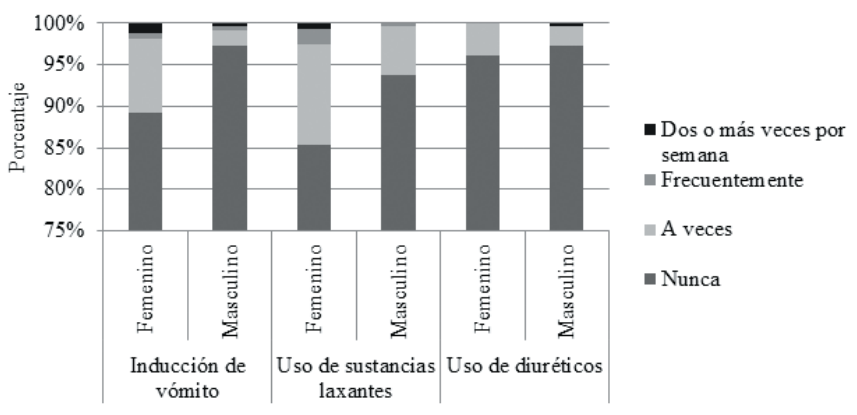

Cuadro 3. Relación estadística de la presencia de conductas de purga entre sí y con la realización de dietas en estudiantes de colegios públicos de Pereira.

\begin{tabular}{lccc}
\hline Conductas relacionadas & $\begin{array}{l}\text { Valor } \\
\text { de } \mathrm{p}^{*}\end{array}$ & $\mathrm{RM}$ & $\mathrm{IC}$ \\
\hline $\begin{array}{l}\text { Inducción de vómito y } \\
\text { realización de dietas }\end{array}$ & 0,001 & 4,05 & $1,668-9,825$ \\
$\begin{array}{l}\text { Uso de laxantes y } \\
\text { realización de dietas }\end{array}$ & 0,002 & 2,87 & $1,440-5,713$ \\
$\begin{array}{l}\text { Laxantes e inducción de } \\
\text { vómito }\end{array}$ & $<0,001$ & 14,5 & $5,828-36,227$ \\
$\begin{array}{l}\text { Laxantes y diuréticos } \\
\text { Inducción de vómito y uso }\end{array}$ & 0,005 & 5,8 & $1,460-23,171$ \\
\hline de diuréticos & & 15,86 & $4,746-53,040$ \\
\hline
\end{tabular}

${ }^{*}$ Prueba de Chi cuadrado de Pearson. IC: Intervalo de Confianza

Los medicamentos más consumidos sin prescripción fueron los anticonceptivos orales por el $11,5 \%(n=18)$ de las mujeres. Los antidepresivos eran consumidos por $1,6 \%$ de los encuestados $(n=6)$, anfetaminas y hormonas tiroideas por el $1,8 \%$ cada uno $(n=7)$, tranquilizantes por $2,1 \%(\mathrm{n}=8)$, modificadores del apetito por $2,4 \%$. $(\mathrm{n}=9)$

\section{Discusión}

Este estudio encontró una proporción significativa de estudiantes con ECA positiva para TCA, sin embargo la prevalencia hallada es la menor reportada hasta el momento en Colombia; esto puede obedecer a diferencias en las edades y distribución de género de las poblaciones incluidas en las diferentes investigaciones. Otros autores han determinado la prevalencia de ECA positiva en el país entre $30 \%$ y $37,08 \%$ en estudiantes de bachillerato de Bucaramanga (11) y Bogotá (9) respectivamente. En estudiantes universitarios de Bogotá la prevalencia fue de $48,8 \%$, siendo significativamente mayor en mujeres, mientras que en una población similar de la ciudad de Cali se encontró ECA positiva en $39,7 \%$ de los participantes (10). Por otro lado, aquellos estudios con poblaciones similares a la incluida en el presente trabajo reportaron, con instrumentos diferentes, los resultados más similares a este $(14,18)$.

Al comparar la prevalencia total de ECA positiva en la presente investigación con prevalencias a nivel internacional en poblaciones similares, esta es notoriamente mayor $(3,21-23)$. Sin embargo, se debe tener en cuenta que encuestas como la Eating Attitudes Test (EAT40) y Eating Disorder Inventory (EDI), que han sido las más utilizadas a nivel mundial, han mostrado sensibilidad, especificidad y valor predictivo positivo más bajos, en especial para el diagnóstico de TCANE (24), que es el más frecuente (6).

Los hallazgos de este estudio soportan la relación entre los TCA, el género femenino (8) y la restricción del número de comidas a la semana $(8,25)$. Por otro lado, este estudio encontró también positividad de ECA y presencia de conductas de purga en hombres, por lo que estos no deben dejarse a un lado para la realización de intervenciones y futuras investigaciones (26).

Las conductas de purga se encontraron en una prevalencia similar a la reportada por otros estudios $(9,10,25)$, y se evidencian como prácticas frecuentes y posiblemente persistentes en la población encuestada, hecho reforzado por la alta coexistencia entre ellas y junto con la realización de dietas para el control del peso que podría indicar que no se trata de hallazgos aislados sino de indicadores de patología establecida (27). La alta prevalencia de realización de dietas requiere atención especial y abordaje multidisciplinario, pues se ha demostrado que los adolescentes que realizan dietas tienen riesgo considerablemente mayor de desarrollar TCA $(6,8)$ y podría afectar el proceso de crecimiento (6).

Es necesario aclarar que la positividad de la ECA que se describe en este estudio debe ser tomada cuidadosamente, ya que a pesar de ser un instrumento con adecuados valores de sensibilidad y especificidad, que fue diseñado y validado para la población del estudio $(11,20)$, el diagnóstico en los trastornos psiquiátricos solo se realiza mediante la entrevista directa, realizada por parte de un profesional capacitado para tal fin, mientras que las escalas diagnósticas no son más que herramientas que pueden indicar la necesidad de una evaluación clínica más detallada (28).

La prevalencia de delgadez encontrada fue medida únicamente mediante la clasificación según IMC, la cual resulta insuficiente para la valoración nutricional de adolescentes, especialmente teniendo en cuenta que las escalas de clasificación del CDC no se ajustan a la población latinoamericana y que deben ser complementadas con otros datos como los pliegues cutáneos (29). Aun así, estos hallazgos hacen meritoria la realización de más estudios para la caracterización del estado nutricional de los adolescentes. De la misma manera, las alteraciones en los ciclos menstruales en la adolescencia no deben ser tomadas como alteraciones patológicas necesariamente, ya que pueden estar relacionadas con el proceso de maduración sexual normal (30) y con el uso de anticonceptivos (31).

Es necesario considerar los TCA como una prioridad para el cuidado de la población adolescente y generar programas de prevención y promoción (32) a nivel regional, que incluyan a toda la población adolescente y preadolescente de colegios y universidades, involucrando aspectos relacionados con la crianza y las competencias parentales, el entorno sociocultural y el enfoque de atención en salud pública desde la infancia.

La presente aproximación permite un mejor entendimiento de los TCA como problema de salud pública a nivel local a pesar de contar con algunas limitantes como el hecho de no incluir estudiantes de colegios privados, la inclusión únicamente de estudiantes de grados 10 y 11 y la valoración nutricional mediante IMC.

De acuerdo a lo anterior, se hace necesaria la ampliación de la información mediante estudios que caractericen mejor algunos aspectos como la relación con las condiciones sociodemográficas, 
imagen corporal y autoestima, los cuales se han visto vinculados estrechamente con estos trastornos $(21,22,33)$. Es necesario considerar a toda la población adolescente como afectada por estos trastornos, ya que existen diferencias significativas entre los presentes hallazgos y los de otros estudios que plantean que el comportamiento de estas patologías podría ser diferente en nuestro medio.

\section{Referencias}

1. Asociación Americana de Psiquiatría. Manual diagnóstico y estadístico de los trastornos mentales. Barcelona: Masson; 2002.

2. Sadock BJ, Sadock VA. Kaplan and Sadock's synopsis of psychiatry: Behavioral sciences/clinical psychiatry: Lippincott Williams \& Wilkins; 2011.

3. Jáuregui Lobera I, Romero Candau J, Bolaños Ríos P, Montes Berriatúa C, Díaz Jaramillo R, Montaña González $M$, et al. Conducta alimentaria e imagen corporal en una muestra de adolescentes de Sevilla. Nutrición Hospitalaria. 2009;24(5):56873.

4. Lavín GM. Trastornos de la conducta alimentaria en adolescentes. ¿Una epidemia? Anales españoles de pediatría. 1998;48(3):22932.

5. Organización Mundial de la Salud. Intervenciones efectivas y opciones de políticas para la prevencion de los Trastornos Mentales. Departamento de Salud Mental y Abuso de Sustancias de la Organización Mundial de la Salud; 2004.

6. Rosen DS. Identification and management of eating disorders in children and adolescents. Pediatrics. 2010;126(6):1240-53.

7. Smink FR, van Hoeken D, Hoek HW. Epidemiology of eating disorders: incidence, prevalence and mortality rates. Current psychiatry reports. 2012;14(4):406-14.

8. Hidalgo $M$, Guemes $M$. Trastornos del comportamiento alimentario: Anorexia y Bulimia. Pediatr Integral. 2008;XII(19):959-72.

9. Avellaneda S. Determinación de la prevalencia de trastornos del comportamiento alimentario en estudiantes de la Pontificia Universidad Javeriana. Bogotá: Universidad Javeriana; 2009.

10. Rodríguez JM, Mina FJ. Prevalencia de factores de riesgo asociados a trastornos del comportamiento alimentario en adolescentes de una institución educativa en Cali, Colombia 2005. Revista Colombiana de Obstetricia y Ginecología. 2008;59(3):180-9.

11. Rueda-Jaimes GE, Cadena Afanador LdP, Díaz-Martínez LA, Ortiz Barajas DP, Pinzón Plata C, Rodríguez Martínez J. Validación de la encuesta de comportamiento alimentario en adolescentes escolarizadas de Bucaramanga, Colombia. Rev colomb de Psiq. 2005;34(3):375-85.

12. Fandiño A, Giraldo S, Martínez C, Aux CP, Espinosa R. Factores asociados con los trastornos de la conducta alimentaria en estudiantes universitarios en Cali, Colombia. Colomb Med. 2007;38(4):344-51.

13. Campo-Arias A, Villamil-Vargas M. Riesgo de trastorno del comportamiento alimentario (TCA) en estudiantes de medicina en Colombia. Revista colombiana de Psiquiatría. 2012;41(2):32839.

14. Castellanos YEL. Frecuencia de los trastornos de la conducta alimentaria en adolescentes en la Ciudad de Bogotá aplicando la escala de tamizaje: the childrens versión of the eating attitudes test (CHEAT). 2012.

15. Sánchez Barrera LF, Manrique Abril FG, Díaz Ospina JM. Evaluación de la conducta alimentaria en estudiantes de Tunja (Boyacá, Colombia) 2012. Revista Hacia la Promoción de la Salud. 2013;18(2):55-65.

16. Forero Rozo MdR, Mejía R, María A, Ardila D, Baena Rivero AL.
Prevalencia de riesgo de los trastornos de la conducta alimentaria en estudiantes de medicina de la Universidad de La Sabana. 2014.

17. Domínguez Ardila AM. Prevalencia de riesgo de trastornos de la conducta alimentaria en estudiantes que inician la vida universitaria. Bogotá: Universidad Javeriana; 2012.

18. Castrillón JJC, Giraldo DL, Guevara J, Losada DL, Meza LM, Narváez DM, et al. Prevalencia de riesgo de trastornos de comportamiento alimentario en una población femenina de estudiantes de secundaria, Manizales, Colombia, 2011. Revista Colombiana de Obstetricia y Ginecología. 2012;63(1):46-56.

19. Ochoa-Orozco SA, Echeverri-Cataño LF, Brito-Carvajal CD, Rodríguez-Morales AJ. Riesgo y uso de escalas diagnósticas en trastornos del comportamiento alimentario (TCA). Revista colombiana de Psiquiatría. 2012;41(4):920-2.

20. Angel L, Vasquez R, Martinez L, Chavarro K, Garcia J. Trastorno del comportamiento alimentario: desarrollo, validez y fiabilidad de una encuesta. Rev Colomb Psiquiat. 2000;29(1):29-48.

21. Palavras MA, Kaio GH, Mari JdJ, Claudino AM. A review of Latin American studies on binge eating disorder. Revista Brasileira de Psiquiatria. 2011;33:s81-s94.

22. Tozun M, Unsal A, Ayranci U, Arslan G. Prevalence of disordered eating and its impact on quality of life among a group of college students in a province of west Turkey. Salud pública de México. 2010;52(3):190-8.

23. Thorsteinsdottir G, Ulfarsdottir L. Eating disorders in college students in Iceland. The European journal of psychiatry. 2008;22(2):107-15.

24. Vetrone G, Cuzzolaro M, Antonozzi I, Garfinkel PE. Screening for eating disorders: false negatives and eating disorders not otherwise specified. The European journal of psychiatry. 2006;20(1):13-20.

25. Olsen do Vale AM, Sansigolo-Kerr LR, Magalhães-Bosi ML. Comportamentos de risco para transtornos do comportamento alimentar entre adolescentes do sexo feminino de diferentes estratos sociais do Nordeste do Brasil. Ciênc saúde coletiva. 2011;16(1):121-32.

26. Strother E, Lemberg R, Stanford SC, Turberville D. Eating disorders in men: underdiagnosed, undertreated, and misunderstood. Eating disorders. 2012;20(5):346-55.

27. Tozzi F, Thornton LM, Klump KL, Fichter MM, Halmi KA, Kaplan AS, et al. Symptom fluctuation in eating disorders: correlates of diagnostic crossover. 2014.

28. Rogers R. Standardizing DSM-IV diagnoses: the clinical applications of structured interviews. Journal of Personality Assessment. 2003;81(3):220-5.

29. Ortiz L. Valoración nutricional en adolescentes. III Composición corporal. Rev Med IMSS. 2002;40(3):223-32.

30. LaCour DE, Long DN, Perlman SE. Dysfunctional uterine bleeding in adolescent females associated with endocrine causes and medical conditions. Journal of pediatric and adolescent gynecology. 2010;23(2):62-70.

31. Kantartzis KL, Sucato GS. Menstrual suppression in the adolescent. Journal of pediatric and adolescent gynecology. 2013;26(3):132-7.

32. Lázaro PR. Prevención primaria de los trastornos de la conducta alimentaria en la adolescencia: papel del profesional de atención primaria. Atención primaria. 2001;27(6):428-31.

33. Quiñones JCG, Restrepo FDLH. Relaciones entre los comportamientos de riesgo psicosociales y la familia en adolescentes de Suba, Bogotá. Revista de Salud Pública. 2014;13(1). 